\title{
On Nondifferentiable and Nonconvex Vector Optimization Problems ${ }^{1}$
}

\author{
Q. H. ANSARI, ${ }^{2} A N D$ J.C. YAO ${ }^{3}$ \\ Communicated by F. G iannessi
}

\begin{abstract}
In this paper, we prove the equivalence among the M inty vector variational-like inequality, Stampacchia vector variational-like inequality, and a nondifferentiable and nonconvex vector optimization problem. By using a fixed-point theorem, we establish also an existence theorem for generalized weakly efficient solutions to the vector optimization problem for nondifferentiable and nonconvex functions.
\end{abstract}

Key Words. Variational-like inequalities, vector optimization problems, generalized solutions, subinvex functions, $\eta$-subdifferential, fixed points.

\section{Introduction and Preliminaries}

In the recent past, vector variational inequalities (VVI) and their generalizations have been used as a tool to solve vector optimization problems (VOP). F or details on VVI and their generalizations, we refer to R ef. 1 and references therein. In $R$ ef. 2, it is shown that a necessary condition for a point to be a so-called weakly efficient solution of a VOP for differentiable functions is that the point be a solution of a suitable VVI. Following the approaches of R ef. 3, L ee studied in R ef. 4 the equivalence between $M$ inty VVI, Stampacchia VVI, both for multivalued maps, and nondifferentiable convex VOP. Inspired by the work of R ef. 3, K omlósi dealt in R ef. 5 with the relationships between M inty VVI, Stampacchia VVI, and their connections with VOP for differentiable functions, while R ef. 6 characterized

\footnotetext{
${ }^{1}$ This research was supported by the $\mathrm{N}$ ational Science Council of the $\mathrm{R}$ epublic of $\mathrm{C}$ hina.

${ }^{2} R$ eader, D epartment of $M$ athematics, A ligarh $M$ uslim U niversity, A ligarh, India; present address: D epartment of A pplied $M$ athematics, $N$ ational Sun $Y$ at-sen $U$ niversity, $K$ aohsiung, Taiwan, ROC.

${ }^{3}$ Professor, Department of Applied Mathematics, $N$ ational Sun $\mathrm{Y}$ at-sen U niversity, K aohsiung, Taiwan, ROC.
} 
generalized monotonicity for multivalued maps in terms of the properties of the associated $M$ inty variational inequalities. In $R$ ef. 7 , the equivalence is established between a vector variational-like inequality (VVLI), a generalized form of the variational inequality studied also in R efs. 8-9, with a multiobjective programming problem for generalized invex functions. In Ref. 10, the equivalence is studied between a VVLI for $\eta$-subdifferential functions and multiobjective optimization problems. The VVLI approach was used in R efs. 11-12 to prove some existence theorems for generalized efficient solutions of nondifferentiable invex VOP. The results in R efs. 1112 are generalizations of existence results established in $R$ ef. 13 for differentiable and convex VOP and in R ef. 14 for differentiable preinvex VOP under the assumption that the constraint set is compact.

In this paper, we prove the equivalence among M inty VVLI, Stampacchia VVLI, both for $\eta$-subdifferentiable functions (R ef. 15), and nondifferentiable nonconvex VOP. We also establish an existence theorem for socalled generalized weakly efficient solutions of nondifferentiable nonconvex VOP by using a fixed-point theorem due to these authors (see R ef. 16).

Let $X$ be a nonempty subset of $\mathbb{R}^{l}$, let $f_{i}: \mathbb{R}^{l} \rightarrow \mathbb{R}, i \in \mid:=\{1, \ldots, l\}$ be real-valued functions, and let $\{C(x): x \in X\}$ be a family of convex cones of $\mathbb{R}^{l}$ each with apex at the origin, such that $\forall x \in X$, int $C(x) \neq \varnothing, C(x) \neq \mathbb{R}^{l}$, and $\mathbb{R}_{+}^{l} \subseteq \mathrm{C}(\mathrm{x})$. We consider the following VOP in the unknown ${ }^{4} y$ :

$$
\min _{x \in X} \operatorname{int}_{C(y)} f(x),
$$

where $f(x):=\left(f_{1}(x), \ldots, f_{l}(x)\right), \min _{\text {int } C(y)}$ marks the vector minimum ${ }^{5}$ with respect to the cone $C(y)$; namely, $y \in X$ is a (global) vector minimum point (vmp) of (1) iff

$$
f(y) \neq_{\text {int } C(y)} f(x), \quad \forall x \in X,
$$

where the inequality means $f(y)-f(x) \notin$ int $C(y)$. Problem (1) is called the generalized weak vector Pareto problem and its vmp's are called generalized weakly efficient points; see R ef. 11 . When $C(y)$ is independent of $y$ and $C=\mathbb{R}_{+}^{l}$, then (1) collapses to the weak vector Pareto problem and its vmp's are called weakly efficient points; see R ef. 17. The term "weakly" comes from the following tradition: when $C(y) \equiv \mathbb{R}_{+}^{l}$, if in (1) int $C$ is replaced by ${ }^{6}$ $\mathbb{R}_{+}^{l} \backslash\{\mathbb{D}\}$, then we have the classic Pareto problem, whose vmp's are called efficient; since the solution set of (1) contains that of Pareto problem, then such a relaxation is named weak.

\footnotetext{
${ }^{4} \mathrm{~N}$ ote that the ordering cone depends on the unknown.

5 int denotes interior.

${ }^{6} \mathrm{~T}$ he symbol $\mathbb{D}$ denotes the origin of the nonnegative orthant, namely $\mathbb{R}_{+}^{l}$.
} 
A differentiable function $g: \mathbb{R}^{l} \rightarrow \mathbb{R}$ is said to be invex (R ef. 18) with respect to (wrt) a given $\eta: \mathbb{R}^{l} \times \mathbb{R}^{l} \rightarrow \mathbb{R}^{l}$ iff

$$
g(x)-g(z) \geq\langle\nabla g(z), \eta(x, z)\rangle, \quad \forall x, z \in \mathbb{R}^{l},
$$

where $\nabla g(z)$ is the gradient vector of $g$ at $z$ and $\langle\cdot, \cdot\rangle$ denotes the scalar product in $\mathbb{R}^{l}$.

It is observed in R ef. 15 that $\nabla g(z)$ is not the only element in $\mathbb{R}^{l}$ which satisfies (3) with the given $\eta$. F or example (R ef. 15), if $g: \mathbb{R} \rightarrow \mathbb{R}$ is a constant function and

$$
\eta(x, z)=(x-z)^{2}
$$

then any $u \in \mathbb{R}$ such that $u \leq 0$ satisfies the following inequality:

$$
g(x)-g(z) \geq\langle u, \eta(x, z)\rangle, \quad \forall x, z \in \mathbb{R} .
$$

The above example also holds for

$$
\eta(x, z)=(x+z)^{2}, \quad \forall x, z \in \mathbb{R},
$$

and for

$$
\eta(x, z)=\left\{\begin{aligned}
a(x-z), & \text { if } x \geq z, \\
-a(x-z), & \text { if } x \leq z,
\end{aligned}\right.
$$

for $a>0$ and $x, z \in \mathbb{R}$.

We note that, when

$$
\eta(x, z)=(x-z)^{2}
$$

then

$$
\eta(x, x)=0 \text { and } \eta(x, z)+\eta(z, x) \neq 0, \text { for } x, z \neq 0 \text {; }
$$

when

$$
\eta(x, z)=(x+z)^{2}
$$

then

$$
\eta(x, x) \neq 0, \quad \text { for } x \neq 0,
$$

and

$$
\eta(x, z)+\eta(z, x) \neq 0, \quad \text { for } x, z \neq 0 \text {; }
$$

when

$$
\eta(x, z)=\left\{\begin{aligned}
a(x-z), & \text { if } x \geq z, \\
-a(x-z), & \text { if } x \leq z,
\end{aligned}\right.
$$


for $a>0 \quad$ and $\quad x, z \in \mathbb{R}$, then

$$
\eta(x, x)=0 \text { and } \eta(x, z)+\eta(z, x)=0 .
$$

This observation motivated us to introduce the following concepts.

A function $g: \mathbb{R}^{l} \rightarrow \mathbb{R}$ is said to be subinvex ${ }^{7}$ at $z \in \mathbb{R}^{l}$ wrt a given map $\eta: \mathbb{R}^{l} \times \mathbb{R}^{l} \rightarrow \mathbb{R}^{l}$ iff $\exists \xi \in \mathbb{R}^{l}$ such that

$$
g(x)-g(z) \geq\langle\xi, \eta(x, z)\rangle, \quad \forall x \in \mathbb{R}^{l} .
$$

Such $\xi$ is called an $\eta$-subgradient of $g$ at $z$, and the set

$$
\partial^{\eta} g(z):=\left\{\xi \in \mathbb{R}^{l}: g(x)-g(z) \geq\langle\xi, \eta(x, z)\rangle, \forall x \in \mathbb{R}^{\prime}\right\}
$$

is called the $\eta$-subdifferential of $g$ at $z$. Therefore, a function is subinvex at a given point wrt $\eta$ iff it has a nonempty $\eta$-subdifferential at that point. The function $g$ is said to be subinvex iff it is subinvex at each $z \in \mathbb{R}^{l}$. It is also noted in R ef. 15 that the set $\partial^{\eta} g(z)$ is not necessarily a singleton, even if $g$ is differentiable, and that it coincides with the subdifferential of convex analysis (R ef. 19) when $g$ is a convex function and $\eta(x, z)=x-z$. Clearly, the class of subinvex functions includes the invex functions with $\xi=\nabla g(z)$.

We give an example of a function $g$ which is subinvex wrt $\eta$ and is discontinuous on an open set $X$.

Let $X=(0,1)$ and define

$$
g(x)= \begin{cases}a, & \text { if } x \in(0,1 / 2), \\ b, & \text { if } x \in[1 / 2,1),\end{cases}
$$

where $a, b>0$ and $a \neq b$ and

$$
\eta(x, z)=\left\{\begin{aligned}
c(x-z), & \text { if } x \geq z, \\
-c(x-z), & \text { if } x \leq z,
\end{aligned}\right.
$$

where $c=|a-b|$. Then, the function $g$ is subinvex wrt $\eta$ and is discontinuous on an open set $X$.

Proposition 1.1. See R ef. 15. If $g: \mathbb{R}^{l} \rightarrow \mathbb{R}$ is subinvex wrt $\eta$ : $\mathbb{R}^{l} \times \mathbb{R}^{l} \rightarrow \mathbb{R}^{l}$, then $\forall z \in \mathbb{R}^{l}, \partial^{\eta} g(z)$ is a nonempty closed convex subset of $\mathbb{R}^{l}$.

A real-valued function $g: \mathbb{R}^{l} \rightarrow \mathbb{R}$ is said to be locally Lipschitz with respect to $\eta: \mathbb{R}^{l} \times \mathbb{R}^{l} \rightarrow \mathbb{R}^{l}$ iff, $\forall \tilde{x} \in \mathbb{R}^{l}$, there exist a neighborhood $N(\tilde{x})$ of $\tilde{x}$

\footnotetext{
${ }^{7}$ The term subinvex has been suggested by Prof. F. G iannessi to replace the term semi-invex used in R ef. 15. Indeed, the term semi-invex would lead one to think that there exist upper and lower semi-invex functions. Instead, what merely happens is that the gradient is replaced by a subgradient.
} 
and a constant $k>0$ such that

$$
|g(x)-g(z)| \leq k\|\eta(x, z)\|, \quad \forall x, z \in N(\tilde{x}) .
$$

If $\eta(x, z)=x-z$, then the concept of locally Lipschitz functions wrt $\eta$ reduces to the definition of locally Lipschitz functions ( $R$ ef. 20 ).

We note that the well-known R ademacher theorem states that a function which is Lipschitz on an open subset of $\mathbb{R}^{l}$ is differentiable almost everywhere (in the sense of the Lebesgue measure) on that subset; see for example R ef. 20. It will be interesting to prove the above result for the class of locally Lipschitz functions with respect to some (fixed) function $\eta$.

Let $T: \mathbb{R}^{l} \rightarrow \mathbb{R}^{l}$ be a multivalued map. Then, $T$ is called locally bounded at $z_{0} \in \mathbb{R}^{l}$ iff there exist a neighborhood $N\left(z_{0}\right)$ of $z_{0}$ and a constant $k>0$ such that the inequality $\|w\| \leq k$ holds $\forall z \in N\left(z_{0}\right)$ and $\forall w \in T(z)$.

$T$ is locally bounded on $\mathbb{R}^{l}$ iff it is locally bounded at each $z \in \mathbb{R}^{l}$.

Proposition 1.2. See R ef. 15 . Let $g: \mathbb{R}^{l} \rightarrow \mathbb{R}$ be subinvex wrt $\eta: \mathbb{R}^{l} \times \mathbb{R}^{l} \rightarrow \mathbb{R}^{l}$, such that $\eta$ is an open map and $\eta(z, z)=\mathbb{O}, \forall z \in \mathbb{R}^{l}$. If $g$ is locally Lipschitz wrt $\eta$, then the multivalued map $\partial^{\eta} g$ is locally bounded on $\mathbb{R}^{l}$.

Proposition 1.3. Let $g: \mathbb{R}^{l} \rightarrow \mathbb{R}$ be subinvex wrt $\eta: \mathbb{R}^{l} \times \mathbb{R}^{l} \rightarrow \mathbb{R}^{l}$, such that $\eta\left(z^{\prime}, z^{\prime \prime}\right)+\eta\left(z^{\prime \prime}, z^{\prime}\right)=\mathbb{O}, \forall z^{\prime}, z^{\prime \prime} \in \mathbb{R}^{l}$. Then, $\partial^{\eta} g$ is an $\eta$-monotone multivalued map; that is, $\forall z^{\prime}, z^{\prime \prime} \in \mathbb{R}^{l}$,

$$
\left\langle\zeta-\xi, \eta\left(z^{\prime}, z^{\prime \prime}\right)\right\rangle \geq 0, \quad \forall \zeta \in \partial^{\eta} g\left(z^{\prime}\right) \text { and } \forall \xi \in \partial^{\eta} g\left(z^{\prime \prime}\right) .
$$

Proof. Let $\zeta \in \partial^{\eta} g\left(z^{\prime}\right)$ and $\xi \in \partial^{\eta} g\left(z^{\prime \prime}\right)$. Then, we have

$$
\begin{array}{ll}
g(x)-g\left(z^{\prime}\right) \geq\left\langle\zeta, \eta\left(x, z^{\prime}\right)\right\rangle, & \forall x \in \mathbb{R}^{l}, \\
g(x)-g\left(z^{\prime \prime}\right) \geq\left\langle\xi, \eta\left(x, z^{\prime \prime}\right)\right\rangle, & \forall x \in \mathbb{R}^{l} .
\end{array}
$$

Put $x=z^{\prime \prime}$ in (4) and $x=z^{\prime}$ in (5), and then add (4) and (5); we get

$$
\left\langle\zeta, \eta\left(z^{\prime \prime}, z^{\prime}\right)\right\rangle+\left\langle\xi, \eta\left(z^{\prime}, z^{\prime \prime}\right)\right\rangle \leq 0 \text {. }
$$

Since

$$
\eta\left(z^{\prime}, z^{\prime \prime}\right)+\eta\left(z^{\prime \prime}, z^{\prime}\right)=\mathbb{O}, \quad \forall z^{\prime}, z^{\prime \prime} \in \mathbb{R}^{l},
$$

we have

$$
\left\langle\zeta-\xi, \eta\left(z^{\prime}, z^{\prime \prime}\right)\right\rangle \geq 0, \quad \forall \zeta \in \partial^{\eta} g\left(z^{\prime}\right) \text { and } \forall \xi \in \partial^{n} g\left(z^{\prime \prime}\right) .
$$


We remark that, if

$$
\eta\left(z^{\prime}, z^{\prime \prime}\right)+\eta\left(z^{\prime \prime}, z^{\prime}\right) \neq \mathbb{D},
$$

then $\partial^{\eta} g$ is not necessarily an $\eta$-monotone multivalued map. For example, if $g$ is a constant function and

$$
\eta\left(z^{\prime}, z^{\prime \prime}\right)=\left(z^{\prime}+z^{\prime \prime}\right)^{2}, \quad \forall z^{\prime}, z^{\prime \prime} \in \mathbb{R} \backslash\{0\},
$$

then it is easy to see that $\partial^{\eta} g$ is not $\eta$-monotone.

If $A$ and $K$ are nonempty subsets of a topological vector space $E$ with $A \subseteq K$, we shall denote by $\operatorname{int}_{K} A$ the interior of $A$ in $K$. If $A$ is a subset of a vector space, conv $A$ denotes the convex hull of $A$. Let $T: X \rightrightarrows Y$ be a multivalued map from a space $X$ to another space $Y$. The graph of $T$, denoted by $G(T)$, is

$$
G(T)=\{(x, u) \in X \times Y: x \in X, u \in T(x)\} .
$$

The inverse $T^{-1}$ of $T$ is a multivalued map from the range of $T$ to $X$, defined by

$$
x \in T^{-1}(u), \quad \text { iff } u \in T(x) .
$$

We shall use the following fixed-point theorem, which generalizes known results in the literature (see $\mathrm{R}$ ef. 16), to prove the existence of generalized weakly efficient solutions to the VOP for nondifferentiable and nonconvex functions.

Theorem 1.1. See R ef. 16. Let $X$ be a nonempty and convex subset of a $\mathrm{H}$ ausdorff topological vector space $E$, and let $S, T: X \rightrightarrows X$ be two multivalued maps such that conv $S(x) \subseteq T(x), S(x) \neq \varnothing, \quad \forall x \in X$, $X=\bigcup\left\{\operatorname{int}_{X} S^{-1}(z): z \in X\right\}$. If $X$ is not compact, we assume that there exist a nonempty compact convex subset $B$ of $X$ and a nonempty compact subset $D$ of $X$ such that, $\forall z \in X \backslash D, \exists \tilde{x} \in B$ such that $z \in \operatorname{int}_{X} S^{-1}(\tilde{x})$. Then, there exists $\bar{z} \in X$ such that $\bar{z} \in T(\bar{z})$.

\section{Vector Variational-Like Inequalities}

Let $X$ be a nonempty subset of $\mathbb{R}^{l}$, and let $f_{i}: \mathbb{R}^{l} \rightarrow \mathbb{R}, i \in \mid:=\{1, \ldots, l\}$, be real-valued functions; let $\eta: X \times X \rightarrow \mathbb{R}^{l}$ be a given map. Then, we consider the following $M$ inty vector variational-like inequality (MVVLI) and Stampacchia vector variational-like inequality (SVVLI):

(M VVLI) find $y \in X$ such that, $\forall x \in X$ and $\forall \zeta_{i} \in \partial^{\eta} f_{i}(x), i \in \mathrm{I}$,

$$
\left(\left\langle\zeta_{1}, \eta(y, x)\right\rangle_{1} \ldots,\left\langle\zeta_{l}, \eta(y, x)\right\rangle\right) \ngtr_{\text {int } C(y)} \mathbb{D} ;
$$


$(\mathrm{SVVLI})_{w}$ find $y \in X$ such that, $\forall x \in X, \exists \xi_{i} \in \partial^{\eta} f_{i}(y), i \in \mid$,

$$
\left(\left\langle\xi_{1}, \eta(y, x)\right\rangle, \ldots,\left\langle\xi_{l}, \eta(y, x)\right\rangle\right) \ngtr_{\text {int } C(y)} \mathbb{D} ;
$$

$(\mathrm{SVVLI})$ s find $y \in X$ such that $\exists \xi_{i} \in \partial^{\eta} f_{i}(y), i \in \mid$, and $\forall x \in X$,

$$
\left(\left\langle\xi_{1}, \eta(y, x)\right\rangle, \ldots,\left\langle\xi_{l}, \eta(y, x)\right\rangle\right) \ngtr_{\text {int } C(y)} \mathbb{D} \text {. }
$$

A solution $y \in X$ of $(\mathrm{SVVLI})_{w}$ is called a weak solution of $(\mathrm{SVVLI})_{s}$; a solution of (SVVLI) $)_{s}$ is called a strong solution of (SVVLI) $)_{w}$. We remark that, in $(\mathrm{SVVLI})_{w}, \xi_{i} \in \partial^{\eta} f_{i}(y), i \in \mathrm{l}$, depend on $x \in X$, but that this is not the case in $(\mathrm{SVVLI})_{s}$. Therefore, every strong solution is a weak solution, but the converse assertion is not true in general. It is sufficient to discuss the weak solution, which is the only need of this paper. F or further details on strong and weak solutions of generalized vector variational inequalities, we refer to R efs. 21-22.

Proposition 2.1. Let $X$ be a nonempty subset of $\mathbb{R}^{l}$, and let $f_{i}: \mathbb{R}^{l} \rightarrow \mathbb{R}$, $i \in \mid$, be subinvex wrt $\eta: X \times X \rightarrow \mathbb{R}^{l}$, such that $\eta(x, z)+\eta(z, x)=\mathbb{D}, \forall x, z \in$ $X$. Then, any solution of (SVVLI) $)_{w}$ is also a solution of (M VVLI).

Proof. Suppose that $y \in X$ is a solution of (SVVLI) $)_{w}$, but not a solution of (M VVLI). Then, $\exists \hat{x} \in X$ and $\hat{\zeta}_{i} \in \partial^{\eta} f_{i}(\hat{x}), i \in l$, such that

$$
\left(\left\langle\hat{\zeta}_{1}, \eta(y, \hat{x})\right\rangle, \ldots,\left\langle\hat{\zeta}_{l}, \eta(y, \hat{x})\right\rangle\right) \in \operatorname{int} C(y) .
$$

From Proposition 1.3, $\partial^{\eta} f_{i}, i \in \mathrm{l}$, are $\eta$-monotone multivalued maps, so that we have

$$
\left\langle\xi_{i}-\zeta_{i}, \eta(y, \hat{x})\right\rangle \geq 0, \quad \forall \zeta_{i} \in \partial^{\eta} f_{i}(\hat{x}) \text { and } \forall \xi_{i} \in \partial^{\eta} f_{i}(y), i \in \mid .
$$

Consequently,

$$
\left\langle\xi_{i}, \eta(y, \hat{x})\right\rangle-\left\langle\zeta_{i}, \eta(y, \hat{x})\right\rangle \in \mathbb{R}_{+}, \quad \forall \zeta_{i} \in \partial^{\eta} f_{i}(\hat{x}) \text { and } \forall \xi_{i} \in \partial^{\eta} f_{i}(y), i \in \mid .
$$

Since

$$
\mathbb{R}_{+}^{l} \subseteq C(y), \quad \forall \xi_{i} \in \partial^{\eta} f_{i}(y), i \in \mid,
$$

we have

$$
\begin{aligned}
\left(\left\langle\xi_{1}, \eta(y, \hat{x})\right\rangle, \ldots,\left\langle\xi_{l}, \eta(y, \hat{x})\right\rangle\right) & \in \operatorname{int} C(y)+\mathbb{R}_{+}^{l} \\
& \subseteq \operatorname{int} C(y)+C(y) \\
& \subseteq \operatorname{int} C(y),
\end{aligned}
$$

which contradicts the assumption. This completes the proof. 
Proposition 2.2. Let $X$ be a nonempty convex subset of $\mathbb{R}^{l}$, and let $\eta$ : $X \times X \rightarrow \mathbb{R}^{l}$ be an open map such that it is continuous in the first argument, affine in the second argument, and $\forall x \in X, \eta(x, x)=\mathbb{0}$. L et $f_{i}: \mathbb{R}^{l} \rightarrow \mathbb{R}, i \in$ I, be subinvex and locally Lipschitz wrt $\eta$. Then, every solution of $(M V V L I)$ is also a solution of (SVVLI) .

Proof. Let $y \in X$ be a solution of (M VVLI). Consider any $x \in X$ and any sequence $\left\{\alpha_{m}\right\} \succ 0$ with $\alpha_{m} \in(0,1]$. Since $X$ is convex,

$$
x_{m}:=\left(1-\alpha_{m}\right) y+\alpha_{m} x \in X .
$$

Since $y \in X$ is a solution of (M VVLI), $\exists \zeta_{i}^{m} \in \partial^{\eta} f_{i}\left(x_{m}\right), i \in \mathrm{I}$, such that

$$
\left(\left\langle\zeta_{1}^{m}, \eta\left(y, x_{m}\right)\right\rangle, \ldots,\left\langle\zeta_{l}^{m}, \eta\left(y, x_{m}\right)\right\rangle\right) \ngtr_{\text {int } C(y)} \mathbb{D} .
$$

Since $\eta(\cdot, \cdot)$ is affine in the second argument and $\eta(x, x)=\mathbb{D}$, we obtain

$$
\alpha_{m}\left(\left\langle\zeta_{1}^{m}, \eta(y, x)\right\rangle, \ldots,\left\langle\zeta_{l}^{m}, \eta(y, x)\right\rangle\right) \ngtr_{\text {int } C(y)} 0 .
$$

We note that

$$
D(y)=\mathbb{R}^{l} \backslash \text { int } C(y)
$$

is a closed cone (see e.g. R ef. 5, pp. 240); therefore, we have

$$
\left(\left\langle\zeta_{1}^{m}, \eta(y, x)\right\rangle, \ldots,\left\langle\zeta_{l}^{m}, \eta(y, x)\right\rangle\right) \neq_{\text {int } C(y)} \mathbb{O} .
$$

F rom Proposition $1.2, \partial^{\eta} f_{i}, i \in \mathrm{I}$, are locally bounded at $y$; hence, there exist a neighborhood $N(y)$ of $y$ and a constant $k>0$ such that, $\forall z \in N(y)$ and $\forall \xi_{i} \in \partial^{\eta} f_{i}(z)$, we have

$$
\left\|\xi_{i}\right\| \leq k, \quad i=1, \ldots, l .
$$

Since $x_{m} \rightarrow y$ as $m \rightarrow \infty, \exists m_{0}$ such that $x_{m} \in N(y), \forall m \geq m_{0}$. Consequently, by (6), we get

$$
\left\|\zeta_{i}^{m}\right\| \leq k, \quad \text { for } m \geq m_{0} .
$$

Without loss of generality, we may assume that the sequence $\left\{\zeta_{i}^{m}\right\}$ converges to $\xi_{i}$. Since $f_{i}, i \in \mid$, are locally Lipschitz wrt $\eta$, and since $\eta$ is continuous in the second variable, it is easy to check that the multivalued map $z \mapsto \partial^{\eta} f_{i}(z)$ has a closed graph, from which it follows that

$$
\xi_{i} \in \partial^{\eta} f_{i}(y), \quad \forall i \in \mid .
$$


Thus, $\forall x \in X, \exists \xi_{i} \in \partial^{\eta} f_{i}(y), i \in \mathrm{l}$, such that

$$
\left(\left\langle\xi_{1}, \eta(y, x)\right\rangle, \ldots,\left\langle\xi_{l}, \eta(y, x)\right\rangle\right) \neq_{\text {int } C(y)} \mathbb{D} .
$$

Hence, $y \in X$ is a solution of (SVVLI) $)_{w}$.

Combining Propositions 2.1 and 2.2, we have the following result.

Theorem 2.1. Let $X$ be a nonempty and convex subset of $\mathbb{R}^{l}$, and let $\eta: X \times X \rightarrow \mathbb{R}^{l}$ be an open map such that it is continuous in the first argument, affine in the second argument, and $\eta(x, z)+\eta(z, x)=\mathbb{O}, \forall x, z \in X$. L et $f_{i}: \mathbb{R}^{l} \rightarrow \mathbb{R}, i \in \mid$, be subinvex and locally Lipschitz wrt $\eta$. Then, $y \in X$ is a solution of (MVVLI) iff it is a solution of $(S V V L I)_{w}$.

We give an example of a function which is subinvex and locally Lipschitz wrt $\eta$.

Let $X=(0,1)$ and define, for each $i \in \mid$,

$$
f_{i}(x)= \begin{cases}a_{i}, & \text { if } x \in(0,1 / 2), \\ b_{i}, & \text { if } x \in[1 / 2,1)\end{cases}
$$

where $a_{i}, b_{i} \geq 0$ and $a_{i} \neq b_{i}$ and

$$
\eta(x, z)=\left\{\begin{aligned}
c(x-z), & \text { if } x \geq z, \\
-c(x-z), & \text { if } x \leq z,
\end{aligned}\right.
$$

where

$$
c=\max _{i \in\rfloor}\left|\mathrm{a}_{i}-\mathrm{b}_{i}\right| .
$$

Then $f_{i}, i \in \mid$, is subinvex and locally Lipschitz wrt $\eta$.

Now, we present the equivalence between (MVVLI) and VOP.

Proposition 2.3. Let $X$ be a nonempty subset of $\mathbb{R}^{l}$, and let $f_{i}: \mathbb{R}^{l} \rightarrow \mathbb{R}$, $i \in \mid$, be subinvex wrt $\eta: X \times X \rightarrow \mathbb{R}^{l}$ such that $\eta(x, z)+\eta(z, x)=\mathbb{O}, \forall x, z \in$ $X$. If $y \in X$ is a solution of (SVVLI) $)_{w}$, then it is a generalized weakly efficient solution of VOP.

Proof. Suppose that $y$ is a solution of $(S V V L I)_{w}$, but not a generalized weakly efficient solution of VOP. Then, $\exists \hat{x} \in X$ such that

$$
f(y)-f(\hat{x}) \geq_{\text {int } C(y)} \mathbb{D}, \quad \text { that is, } f(y)-f(\hat{x}) \in \operatorname{int} C(y) .
$$


Since $\mathbb{R}_{+}^{l} \subseteq C(y)$ and $f_{i}, i \in \mathrm{l}$, are subinvex wrt $\eta, \forall \xi_{i} \in \partial^{\eta} f_{i}(y), i \in \mathrm{I}$, we have

$$
\begin{aligned}
\left(\left\langle\xi_{1}, \eta(y, \hat{x})\right\rangle, \ldots,\left\langle\xi_{l}, \eta(y, \hat{x})\right\rangle\right) & \in f(y)-f(\hat{x})+\mathbb{R}_{+}^{l} \\
& \subseteq \operatorname{int} C(y)+C(y) \\
& \subseteq \operatorname{int} C(y),
\end{aligned}
$$

which contradicts our supposition. This completes the proof.

Proposition 2.4. Let $X$ be a nonempty subset of $\mathbb{R}^{l}$, and let $\eta: X \times X \rightarrow \mathbb{R}^{l}$ be a map. Let $f_{i}: \mathbb{R}^{l} \rightarrow \mathbb{R}, i \in \mid$, be subinvex wrt $\eta$. Then, any generalized weakly efficient solution of VOP is also a solution of (M VVLI).

Proof. A ssume that $y \in X$ is a generalized weakly efficient solution of VOP, but not a solution of (M V VLI). Then, $\exists \hat{x} \in X$ and $\zeta_{i} \in \partial^{\eta} f_{i}(\hat{x}), i \in \mid$, such that

$$
\left(\left\langle\zeta_{1}, \eta(y, \hat{x}), \ldots,\left\langle\zeta_{l}, \eta(y, \hat{x})\right\rangle\right) \geq_{\text {int } C(y)} \mathbb{D} .\right.
$$

By subinvexity of $f_{i}, i \in \mid$, wrt $\eta$, we have

$$
f_{i}(y)-f_{i}(\hat{x})-\left\langle\zeta_{i}, \eta(y, \hat{x})\right\rangle \in \mathbb{R}_{+},
$$

and thus,

$$
\begin{aligned}
& \left(f_{1}(y)-f_{1}(\hat{x}), \ldots, f_{l}(y)-f_{l}(\hat{x})\right) \\
& -\left(\left\langle\zeta_{1}, \eta(y, \hat{x})\right\rangle_{1} \ldots,\left\langle\zeta_{l}, \eta(y, \hat{x})\right\rangle\right) \in \mathbb{R}_{+}^{l} .
\end{aligned}
$$

F rom (7)-(8), we then have

$$
\left(f_{1}(y)-f_{1}(\hat{x}), \ldots, f_{l}(y)-f_{l}(\hat{x})\right) \geq_{\operatorname{int} C(y)} \mathbb{D},
$$

which contradicts our assumption. H ence, the result is proved. result.

F rom Theorem 2.1 and Propositions 2.3 and 2.4, we have the following

Theorem 2.2. Let $X$ be a nonempty and convex subset of $\mathbb{R}^{l}$, and let $\eta: X \times X \rightarrow \mathbb{R}^{l}$ be an open map such that it is continuous in the first argument, affine in the second argument, and $\eta(x, z)+\eta(z, x)=\mathbb{O}, \forall x, z \in X$. L et $f_{i}: \mathbb{R}^{l} \rightarrow \mathbb{R}, i \in \mid$, be subinvex and locally Lipschitz wrt $\eta$. Then, $y \in X$ is a generalized weakly efficient solution of VOP iff it is a solution of (M VVLI). 


\section{Existence Result for Vector Optimization Problems}

In this section, we prove the existence of generalized weakly efficient solutions to the nondifferentiable and nonconvex VOP by using a fixedpoint theorem due to these authors (R ef. 16).

Theorem 3.1. Let $X$ be a nonempty and convex subset of $\mathbb{R}^{l}$, and let $\eta: X \times X \rightarrow \mathbb{R}^{l}$ be an open map such that it is continuous in the first argument, affine in the second argument, and $\eta(x, z)+\eta(z, x)=\mathbb{O}, \forall x, z \in X$. L et $f_{i}: \mathbb{R}^{l} \rightarrow \mathbb{R}, i \in \mid$, be subinvex wrt $\eta$, and let $W: K \rightrightarrows \mathbb{R}^{l}$ be a multivalued map defined by $W(x)=\mathbb{R}^{l} \backslash$ int $C(x), \forall x \in X$, such that its graph is closed. A ssume that there exist a nonempty compact convex subset $B$ of $X$ and a nonempty compact subset $D$ of $X$ such that, $\forall z \in X \backslash D, \exists \tilde{x} \in B$ and $\zeta_{i} \in \partial^{\eta} f_{i}(\tilde{x}), i \in \mid$, such that

$$
\left(\left\langle\zeta_{1}, \eta(z, \tilde{x})\right\rangle, \ldots,\left\langle\zeta_{l}, \eta(z, \tilde{x})\right\rangle\right) \geq_{\operatorname{int} C(z)} \mathbb{D} .
$$

Then, VOP has a generalized weakly efficient solution.

Proof. For the sake of simplicity, we denote $\partial^{\eta} f_{1}(x) \times \cdots \times \partial^{\eta} f_{l}(x)$ by $\partial^{\eta} f(x)$ and $\left(\left\langle\xi_{1}, \eta(z, x)\right\rangle, \ldots,\left\langle\xi_{l}, \eta(z, x)\right\rangle\right)$ by $\langle\xi, \eta(z, x)\rangle$, where $\xi=$ $\left(\xi_{1}, \ldots, \xi_{l}\right) \in \mathbb{R}^{l \times l}$. We define a multivalued map $\left.G: X \rightrightarrows\right) X$ by

$$
G(x)=\left\{z \in X: \forall \zeta \in \partial^{\eta} f(x) \text { s.t. }\langle\zeta, \eta(z, x)\rangle \ngtr_{\text {int } C(z)} \mathbb{D}\right\}, \quad \forall x \in X .
$$

Then, $\forall x \in X, G(x)$ is closed in $X$. Indeed, let $\left\{z_{m}\right\}$ be a sequence in $G(x)$ such that $z_{m} \rightarrow z \in X$. Then, $\forall \zeta \in \partial^{\eta} f(x)$, we have

$$
\left\langle\zeta, \eta\left(z_{m}, x\right)\right\rangle \ngtr_{\text {int } C\left(z_{m}\right)} \mathbb{D} \text {, i.e., }\left\langle\zeta, \eta\left(z_{m}, x\right)\right\rangle \in W\left(z_{m}\right) .
$$

Since $\eta(\cdot, x)$ and the pairing $\langle\cdot, \cdot\rangle$ are continuous, we have

$$
\left\langle\zeta, \eta\left(z_{m}, x\right)\right\rangle \rightarrow\langle\zeta, \eta(z, x)\rangle \in W(z),
$$

because the graph of $W$ is closed. Hence,

$$
\langle\zeta, \eta(z, x)\rangle \ngtr_{\text {int } C(z)} \mathbb{D}, \quad \forall \zeta \in \partial^{\eta} f(x) .
$$

Hence $z \in G(x)$, and thus $G(x)$ is closed in $X$.

In view of Theorem 2.2, it is sufficient to prove the existence of a solution of (MVVLI). Suppose that (MVVLI) does not have any solution. Then, $\forall z \in X$, the set

$\left\{x \in X: \exists \zeta \in \partial^{\eta} f(x)\right.$ s.t. $\left.\langle\zeta, \eta(z, x)\rangle \geq_{\text {int } C(z)} \mathbb{D}\right\}=\{x \in X: z \notin G(x)\} \neq \varnothing$. 
Now, we define two multivalued maps $S, T: X \rightrightarrows X$ by

$$
\begin{aligned}
& S(z)=\left\{x \in X: \exists \zeta \in \partial^{\eta} f(x) \text { s.t. }\langle\zeta, \eta(z, x)\rangle \geq_{\text {int } C(z)} \mathbb{D}\right\}, \\
& T(z)=\left\{x \in X: \forall \xi \in \partial^{\eta} f(z) \text { s.t. }\langle\xi, \eta(z, x)\rangle \geq_{\text {int } C(z)} \mathbb{D}\right\}, \quad \forall z \in X .
\end{aligned}
$$

Then clearly, $\forall z \in X$,

$$
S(z) \neq \varnothing \text {. }
$$

Let $\left\{x_{1}, \ldots, x_{m}\right\}$ be a finite subset of $S(z)$. Then, $\exists \zeta_{i} \in \partial^{\eta} f\left(x_{i}\right), i=1, \ldots, m$, s.t.

$$
\left\langle\zeta_{i}, \eta\left(z, x_{i}\right)\right\rangle \geq_{\text {int } C(z)} \mathbb{D}, \quad \forall i=1, \ldots, m .
$$

Since $\partial^{\eta} f$ is an $\eta$-monotone multivalued map, we have, $\forall i=1, \ldots, m$, $\left\langle\zeta_{i}, \eta\left(x_{i}, z\right)\right\rangle-\left\langle\xi, \eta\left(x_{i}, z\right)\right\rangle \in \mathbb{R}_{+}^{l} \subseteq C(z), \quad \forall \zeta_{i} \in \partial^{\eta} f\left(x_{i}\right)$ and $\forall \xi \in \partial^{\eta} f(z)$.

Since

$$
\eta(x, z)+\eta(z, x)=\mathbb{O}, \quad \forall x, z \in X,
$$

we have

$\left\langle\xi, \eta\left(z, x_{i}\right)\right\rangle-\left\langle\zeta_{i}, \eta\left(z, x_{i}\right)\right\rangle \subseteq C(z), \quad \forall \zeta_{i} \in \partial^{\eta} f\left(x_{i}\right)$ and $\forall \xi \in \partial^{\eta} f(z)$.

F rom (9)-(10), we get, $\forall i=1, \ldots, m$,

$$
\begin{aligned}
\left\langle\xi, \eta\left(z, x_{i}\right)\right\rangle & \in\left\langle\zeta_{i}, \eta\left(z, x_{i}\right)\right\rangle+C(z) \\
& \subseteq \operatorname{int} C(z)+C(z) \\
& \subseteq \operatorname{int} C(z) .
\end{aligned}
$$

Let $\alpha_{i} \geq 0$ such that $\sum_{i=1}^{m} \alpha_{i}=1$. Since $C(z)$ is a convex cone, we have

$\left\langle\xi, \alpha_{1} \eta\left(z, x_{1}\right)\right\rangle+\cdots+\left\langle\xi, \alpha_{m} \eta\left(z, x_{m}\right)\right\rangle \in \operatorname{int} C(z)$.

Since $\eta(x, \cdot)$ is affine, we get

$$
\langle\xi, \eta(z, \hat{x})\rangle \in \operatorname{int} C(z),
$$

where $\hat{x}=\sum_{i=1}^{m} \alpha_{i} x_{i}$. H ence, $\hat{x} \in T(z)$; therefore,

$$
\text { conv } S(z) \subseteq T(z), \quad \forall z \in X .
$$

Since $G(x)$ is closed in $X, S^{-1}(x)=[G(x)]^{c}$ [the complement of $G(x)$ in $\left.X\right]$ is open in $X$, and hence

$$
\text { int }_{X} S^{-1}(x)=S^{-1}(x) \text {. }
$$

Since, $\forall z \in X, S(z) \neq \varnothing$, we have

$$
X=\bigcup_{x \in X} S^{-1}(x)=\bigcup_{x \in X} \operatorname{int}_{X} S^{-1}(x) .
$$


Now, for each $z \in X \backslash D, \exists \tilde{x} \in B$ and $\zeta \in \partial^{\eta} f(\tilde{x})$ such that

$$
\langle\zeta, \eta(\tilde{x}, z)\rangle \geq_{\operatorname{int} C(z)} \mathbb{D},
$$

and hence,

$$
z \in S^{-1}(\tilde{x})=\operatorname{int}_{X} S^{-1}(\tilde{x}) .
$$

Thus, $S$ and $T$ satisfy all the conditions of Theorem 1.1. Therefore from Theorem 1.1, there exists $\bar{z} \in X$ such that $\bar{z} \in T(\bar{z})$, that is, $\forall \xi \in$ $\partial^{\eta} f(\bar{z})$ s.t. $\langle\xi, \eta(\bar{z}, \bar{z})\rangle \geq_{\text {int } C(\bar{z})} \mathbb{D}$. Since

$$
\eta(z, x)+\eta(x, z)=0, \quad \forall x, z \in K,
$$

we have $\eta(\bar{z}, \bar{z})=\mathbb{O}$, and thus

$$
\langle\xi, \eta(\bar{z}, \bar{z})\rangle=\mathbb{D} \in \operatorname{int} C(\bar{z}),
$$

which contradicts the fact that $\mathbb{D} \notin$ int $C(z)$. This completes the proof.

\section{References}

1. GiAnnessI, F. (Editor), Vector Variational Inequalities and Vector Equilibria: Mathematical Theories, Kluwer Academic Publishers, Dordrecht, Holland, 2000.

2. Lee, G. M., Kim, D. S., LeE, B. S., and Yen, N. D., Vector Variational Inequality as a Tool for Studying Vector Optimization Problems, N onlinear A nalysis: Theory, M ethods, and A pplications, Vol. 34, pp. 745-765, 1998.

3. Giannessi, F., On Minty Variational Principle, $\mathrm{N}$ ew Trends in $\mathrm{M}$ athematical Programming, Edited by F. Giannessi, S. K omlósi, and T. Rapcsák, K luwer A cademic Publishers, D ordrecht, H olland, pp. 93-99, 1998.

4. Le e, G. M ., On Relations between Vector Variational Inequality and Vector Optimization Problem, Progress in Optimization, II : Contributions from A ustralasia, Edited by X. Q. Y ang, A. I. M ees, M. E. F isher, and L. S. J ennings, K luwer A cademic Publishers, D ordrecht, Holland, 2000 (in press).

5. Komlósı, S., On the Stampacchia and Minty Variational Inequalities, Generalized Convexity and Optimization for Economic and Financial Decisions, Edited by G. G iorgi and F. R ossi, Pitagora Editrice, Bologna, Italy, pp. 231260, 1999.

6. John, R., A Note on Minty Variational Inequalities and Generalized Monotonicity, Discussion Paper A-596, U niversity of Bonn, 1999.

7. Y ANG, X. Q., Generalized Convex Functions and Vector Variational Inequalities, Journal of Optimization Theory and A pplications, Vol. 79, pp. 563-580, 1993.

8. LIN, L. J., Prevariational Inequalities, Bulletin of the A ustralian M athematical Society, Vol. 53, pp. 63-70, 1996. 
9. Siddiqu, A. H., Ansari, Q. H., and Ahmad, R., On Vector Variational-Like Inequalities, Indian Journal of Pure and Applied M athematics, Vol. 28, pp. 1009-1016, 1997.

10. Ansari, Q. H., and Siddiqı, A. H., A Generalized Vector Variational-Like Inequality and Optimization over an Efficient Set, F unctional A nalysis with Current A pplications in Science, Engineering, and Industry, Edited by M. Brokate and $\mathrm{A}$. H. Siddiqi, Pitman R esearch $\mathrm{N}$ otes in $\mathrm{M}$ athematics, L ongman, Essex, England, Vol. 377, pp. 177-191, 1998.

11. L E E, G. M ., K IM, D. S., and K U K, H ., Existence of Solutions for Vector Optimization Problems, Journal of $M$ athematical A nalysis and Applications, Vol. 220, pp. 90-98, 1998.

12. Lee, G. M ., LeE, B. S., and ChAn G, S. S., On Vector Quasivariational Inequalities, J ournal of M athematical A nalysis and A pplications, Vol. 203, pp. 626-638, 1996.

13. Chen, G. Y., and Craven, B. D., A Vector Variational Inequality and Optimization over an Efficient Set, ZOR -M ethods and M odels in Operations R esearch, Vol. 3, pp. 1-12, 1990.

14. K Azm I, K. R., Existence of Solutions for Vector Optimization, Applied M athematics L etters, Vol. 9, pp. 19-22, 1996.

15. Dutta, J., Vetrivel, V., and Nanda, S., Semi-Invex Functions and Their Subdifferentials, Bulletin of the A ustralian M athematical Society, Vol. 56, pp. 385393, 1997.

16. AnsA RI, Q. H., and Y A O, J. C., A Fixed-Point Theorem and Its Applications to the System of Variational Inequalities, Bulletin of the A ustralian M athematical Society, Vol. 59, pp. 435-444, 1999.

17. Sawaragi, Y., Nakayama, H., and Tanino, T., Theory of Multiobjective Optimization, A cademic Press, N ew Y ork, NY, 1985.

18. Hanson, M. A., On the Sufficiency of Kuhn-Tucker Conditions, Journal of M athematical A nalysis and A pplications, Vol. 80, pp. 545-550, 1981.

19. Rock a fellar, R. T., Convex Analysis, Princeton U niversity Press, Princeton, N ew J ersey, 1970.

20. Clarke, F. H., Optimization and Nonsmooth Analysis, SIAM, Philadelphia, Pennsylvania, 1990.

21. K ON N OV, I. V., and Y A O, J. C., On the Generalized Vector Variational Inequality Problem, J ournal of $M$ athematical A nalysis and A pplications, Vol. 206, pp. 4258, 1997.

22. AnsA RI, Q. H., and Y A O, J. C., On Strong Solutions of the Generalized Implicit Vector Variational Problem, Advances in Nonlinear Variational Inequalities, Vol. 2, pp. 1-10, 1999. 